\title{
Endothelial cell-expressed Tim-3 facilitates metastasis of melanoma cells by activating the NF- $\mathrm{kB}$ pathway
}

\author{
FENG-HUA WU, YE YUAN, DONG LI, ZHANG LEI, CHUAN-WANG SONG, \\ YAN-YAN LIU, BO LI, BO HUANG, ZUO-HUA FENG and GUI-MEI ZHANG \\ Department of Biochemistry and Molecular Biology, Tongji Medical College, \\ Huazhong University of Science and Technology, Wuhan 430030, P.R. China
}

Received March 16, 2010; Accepted June 9, 2010

DOI: 10.3892/or_00000909

\begin{abstract}
T cell immunoglobulin and mucin domain-3 (Tim-3) is originally recognized as a receptor of Th1 cells. We found that Tim-3 could be expressed in endothelial cells after stimulation with tumor cell-released TLR4 ligand. Tim-3 expressed by endothelial cells does not function as the receptor of galectin-9, but mediates the interaction of endothelial cells with tumor cells. The engagement of endothelial cellexpressed Tim-3 with a non-galectin 9 putative receptor on B16 melanoma cells could trigger the NF- $\kappa$ B signaling pathway in B16 cells. The activated NF- $\mathrm{B}$ not only promoted the proliferation of B16 cells, but also enhanced apoptosis resistance of B16 cells by up-regulating Bcl-2 and Bcl-xL and down-regulating Bax. Consistently, Tim-3 facilitated the survival of B16 cells in the blood stream, arrested in the lung and following invasion, resulted in more metastatic nodules in the lung. These findings suggest that endothelial cell-expressed Tim-3 increases tumor cell metastatic potential by facilitating tumor cell intravasation, survival in blood stream and extravasation. Thus, antiinflammation or blockade of Tim-3 may contribute to the prevention of metastasis.
\end{abstract}

\section{Introduction}

Chronic inflammation has long been known to be braided with tumor growth, and considered to contribute to tumor metastasis. Toll-like receptor (TLR) signaling is a key event to mediate inflammation. TLRs can be expressed not only in tumor-infiltrating leukocytes but also in other stromal cells, such as endothelial cells. Importantly, endothelial cells also contribute to tumor inflammation and metastasis (1-3). TLR4 expressed in endothelial cells can be activated by engaging with TLR4 ligands such as LPS or high mobility group box 1

Correspondence to: Dr Gui-Mei Zhang, Department of Biochemistry and Molecular Biology, Tongji Medical College, Huazhong University of Science and Technology, Wuhan 430030, P.R. China E-mail: zhanggm58@163.com

Key words: endothelial cells, Tim-3, NF-кB pathway, tumor metastasis
(HMGB1) $(4,5)$. Subsequently, various inflammatory factors are induced in endothelial cells (5-7). Therefore, in parallel with the development of tumor inflammation, the phenotype and function of tumor-associated endothelial cells may be modified by TLR4 ligands. Regardless of the intensive study of tumor-associated endothelial cells, the involvement of endothelial cells in tumor inflammation and metastasis is still incompletely elucidated.

T cell immunoglobulin and mucin domain-3 (Tim-3) was originally recognized as a Th1-specific receptor that regulates Th1 responses and maintains immune homeostasis and tolerance $(8,9)$. However, recent studies have demonstrated the expression of Tim-3 in other types of cells (10-12). Moreover, the up-regulation of Tim-3 has been confirmed in different inflammatory diseases (13-15). In this study, we found that Tim-3 could mediate the communication between endothelial cells and tumor cells. Using a mouse melanoma model, we identified that the expression of Tim-3 in tumor-associated endothelial cells could be induced by TLR4 ligand. Tim-3 in turn promoted tumor growth and metastasis. These findings provide new insight into the molecular basis for the interaction of tumor cells with endothelial cells in the process of tumor metastasis.

\section{Materials and methods}

Mice and cell lines. C57BL/6 mice were purchased from Center of Experimental Animals of Chinese Academy of Medical Science (Beijing, China). Melanoma B16-F1 cell line, Chinese hamster ovary cell line $(\mathrm{CHO})$, and murine microvascular endothelial cell line bEnd.3 were purchased from CCTCC (Wuhan, China) and ATCC (Manassas, VA), respectively, and cultured according to their guidelines.

Immunohistochemistry. Mice were inoculated by injection of $1 \times 10^{5}$ tumor cells into right hind thigh muscle. Fifteen days after inoculation, tumors were surgically excised for the preparation of sections. The sections were fixed and stained with rat anti-mouse Tim-3 mAb, biotinylated goat anti-rat $\mathrm{IgG}$, and streptavidin-conjugated horseradish peroxidase (eBioscience, San Diego, CA). All animal experiments were conducted according to our institutional guidelines.

Analysis of Tim-3 expressed in endothelial cells. Tumors or lung tissues were digested with collagenase and hyaluronidase. 
The enriched endothelial cells were obtained from the dissociated cells by Percoll density gradient centrifugation as described previously (16). In other experiments, bEnd.3 cells were stimulated with HMGB1 $(100 \mathrm{ng} / \mathrm{ml}, \mathrm{R} \& \mathrm{D}$ Systems, Minneapolis, MN) for $48 \mathrm{~h}$ in absence or presence of AP-1 inhibitor Tanshinone IIA ( $2 \mu \mathrm{M}$, Biomol, Plymouth Meeting, PA) or NF- $\kappa$ B inhibitor $(0.2 \mu \mathrm{M}$, Calbiochem, San Diego, $\mathrm{CA})$. The cells were analyzed by flow cytometry.

Flow cytometry. For the detection of Tim-3, the cells were stained with PE-conjugated anti-mouse Tim-3 (eBiosciences) or isotype control Rat $\operatorname{IgG1}$, and then analyzed by flow cytometry. In the indicated cases, the cells were co-stained with APC-conjugated anti-mouse TLR4 and FITC-conjugated anti-mouse CD31 antibodies and corresponding isotype antibodies (eBioscience). For the detection of Tim-3-binding putative receptor, the cells were incubated with Tim-3-Fc (eBioscience) or human IgG (isotype control) and then stained with PE-conjugated goat anti-human IgG-Fc (eBioscience) for flow cytometric analysis.

Analysis of gene expression by conventional RT-PCR and real-time RT-PCR. Total RNA was extracted from cells with TRIzol reagent (Invitrogen) according to the manufacturer's instructions. The mRNA level was determined by RT-PCR or real-time RT-PCR as described previously (17). Primers were designed with the Oligo Primer Analysis 4.0 software and the sequences were blasted (http://www.ncbi.nlm.nih.gov/ BLAST/).

Western blot analysis. Western blot assay was done as described previously (17). All antibodies were purchased from Santa Cruz Biotechnology (Santa Cruz, CA).

Assay of activity of $N F-\kappa B$. The nuclear extract was prepared with Nuclear Extraction kit (Millipore, Billerica, MA). The

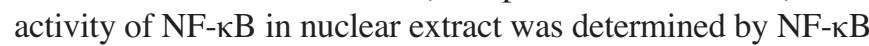
Assay kit (Millipore) according to the manufacturer's protocol.

Cell transfection. Eukaryotic expression vector pTim-3 carrying the cDNA encoding full-length mouse Tim-3 was preserved in the laboratory (18). CHO cells were transfected with pTim-3 or pcDNA3.1 with FuGene 6 transfection reagent (Roche, Indianapolis, IN) according to the manufacturer's protocol. After selection with G418, two clones were obtained, which were designated as Tim-3-CHO (CHO/pTim-3) and control-CHO $(\mathrm{CHO} / \mathrm{pcDNA} 3.1)$ respectively.

Cell fixation. Tim-3-CHO cells, Tim- $3^{+}$bEnd cells (stimulated with $1 \mu \mathrm{g} / \mathrm{ml}$ LPS for $48 \mathrm{~h}$ ) or corresponding control cells were plated in culture plate. Twenty-four hours later, the cell confluence was $>90 \%$. Then, the culture medium was discarded and the cells were fixed with $3 \%$ glutaraldehyde at RT for 15 min and then washed three times.

Preparation of recombinant galectin 9. Expression plasmid of galectin 9 was generated in vector pTrcHis2A (Invitrogen, Carlsbad, CA) and was transformed into BL21 Escherichia coli (Invitrogen). Protein induction and purification were done as described previously (19). Purified galectin-9 was applied to Detoxi-Gel (Pierce, Rockford, IL) for removal of endotoxin.
Proliferation assay. B16 cells were seeded in 96-well plate $\left(5 \times 10^{3}\right.$ per well) containing pre-fixed cells, and cultured for $48 \mathrm{~h}$ in the presence or absence of anti-Tim-3 blocking antibody $(10 \mu \mathrm{g} / \mathrm{ml})$. In the indicated cases, the cells were cultured in the presence of NF-kB inhibitor. The proliferation assay was performed with MTT Cell Proliferation kit (Roche Diagnostics, IN) according to the manufacturer's instructions.

Apoptosis assay. bEnd. 3 cells were cultured for $48 \mathrm{~h}$ in absence or presence of galectin-9 $(1 \mu \mathrm{M})$, then stained with PEAnnexin V (BD Biosciences, San Diego, CA) and analyzed by flow cytometry. Spleen T cells activated with Con A for $48 \mathrm{~h}$ were used as control. In other experiments, B16 cells were cultured in the presence of pre-fixed cells for $48 \mathrm{~h}$, and then irradiated with UVB $\left(200 \mathrm{~J} / \mathrm{m}^{2}\right)$ or treated with mitomycin C (MMC, $10 \mu \mathrm{g} / \mathrm{ml}$ ) for $12 \mathrm{~h}$. The apoptosis of cells were analyzed by flow cytometry. For the assay of B16 cell apoptosis in vivo, B16 cells were labeled with CFSE and injected into mice $\left(2 \times 10^{6}\right.$ per mouse) via tail vein. Five and twelve hours later, the whole blood was collected from mice. After lysis of RBC, the left cells were analyzed by flow cytometry. The proportion of apoptotic B16 cells were calculated by Annexin- $\mathrm{V}^{+} \mathrm{CFSE}^{+}$cells/total $\mathrm{CFSE}^{+}$cells .

Adhesion assay. B16 cells were added to 6-well plate containing pre-fixed Tim- $3^{+}$cells in absence or presence of antiTim-3 antibody. After $2-\mathrm{h}$ incubation at $37^{\circ} \mathrm{C}$, non-adherent cells were harvested. Then, adherent cells were harvested by treatment with trypsin. The percentage of adherent cells was calculated.

Assay of tumor cell arrest and metastasis in the lung. B16 cells were labeled with CFSE and injected into mice $\left(5 \times 10^{5}\right.$ per mouse) via tail vein. Lungs were harvested from mice 5 and $24 \mathrm{~h}$ after the injection. Frozen sections were prepared and analyzed by fluorescence microscopy. Fluorescent spots were counted from 20 randomly chosen fields in the sections of each mouse. For the analysis of tumor metastasis, B16 cells were injected into mice via tail vein. The mice were sacrificed on day 15 for counting tumor nodules on the surface of the lung.

Data analysis. Results were expressed as mean value \pm SD and interpreted by one-way ANOVA. Differences were considered to be statistically significant at $\mathrm{P}<0.05$.

\section{Results}

HMGB1 induces Tim-3 expression in endothelial cells. To explore the possible link of Tim-3 to tumor, we first stained tumor tissues to analyze the cellular source of Tim-3 in a murine melanoma model. The result showed that blood vessel was positively stained (Fig. 1A). The analysis of flow cytometry further confirmed that endothelial cells $\left(\mathrm{CD} 31^{+}\right)$in tumor indeed expressed Tim-3 (Fig. 1B). Tim-3 expression in tumor was increased along with tumor progression (Fig. 1C). Coincidently, the amount of HMGB1 in the interstitial fluid of tumor was also gradually increased (Fig. 1D). We then investigated the effect of HMGB1 on Tim-3 expression by using a mouse microvascular endothelial cell line bEnd.3. 
A

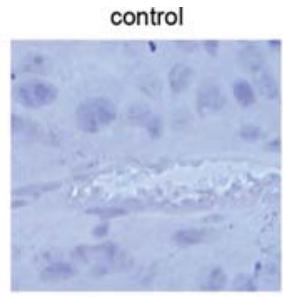

d10 $\quad$ d15 d20

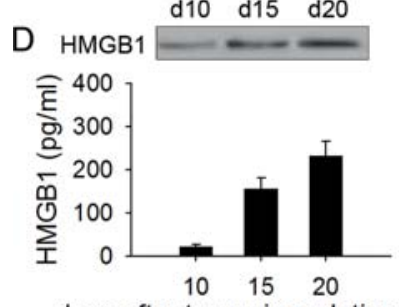

days after tumor inoculation
anti-Tim-3

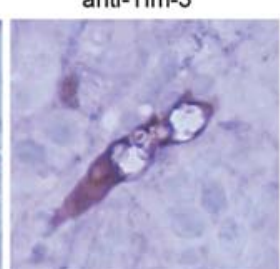

E

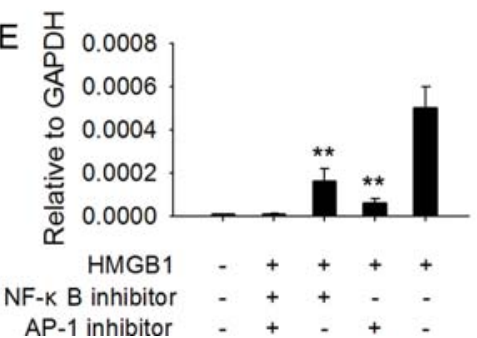

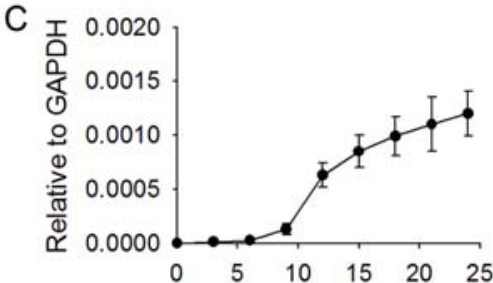

days after tumor inoculatioin

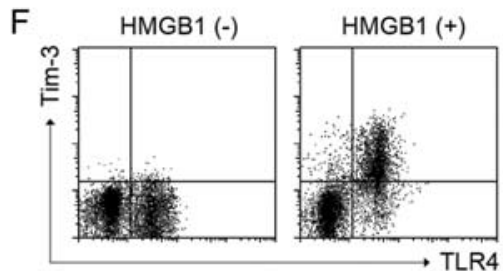

Figure 1. HMGB1 induces expression of Tim-3 in endothelial cells. (A and B) Detection of Tim-3 in endothelial cells. The sections of tumor tissues were analyzed by immunohistochemical staining (A). Enriched endothelial cells from tumor tissues were analyzed by flow cytometry (B). (C) Real-time RT-PCR analysis of Tim-3 expression in tumor on the indicated day after tumor inoculation. (D) Detection of HMGB1 in tumor. Tumor tissues were digested with collagenase and hyaluronidase on the indicated day after tumor inoculation. HMGB1 in the interstitial liquid of tumor was detected by Western blotting and ELISA. (E and F) HMGB1 induces Tim-3 expression. bEnd.3 cells were stimulated with HMGB1 in absence or presence of NF-kB inhibitor and/or AP-1 inhibitor for $48 \mathrm{~h}$. The transcription of Tim-3 gene was detected by real-time RT-PCR (E). ${ }^{* *} \mathrm{P}<0.01$, compared with HMGB1 group. bEnd.3 cells cultured in absence or presence of HMGB1 were analyzed by flow cytometry $(\mathrm{F})$.
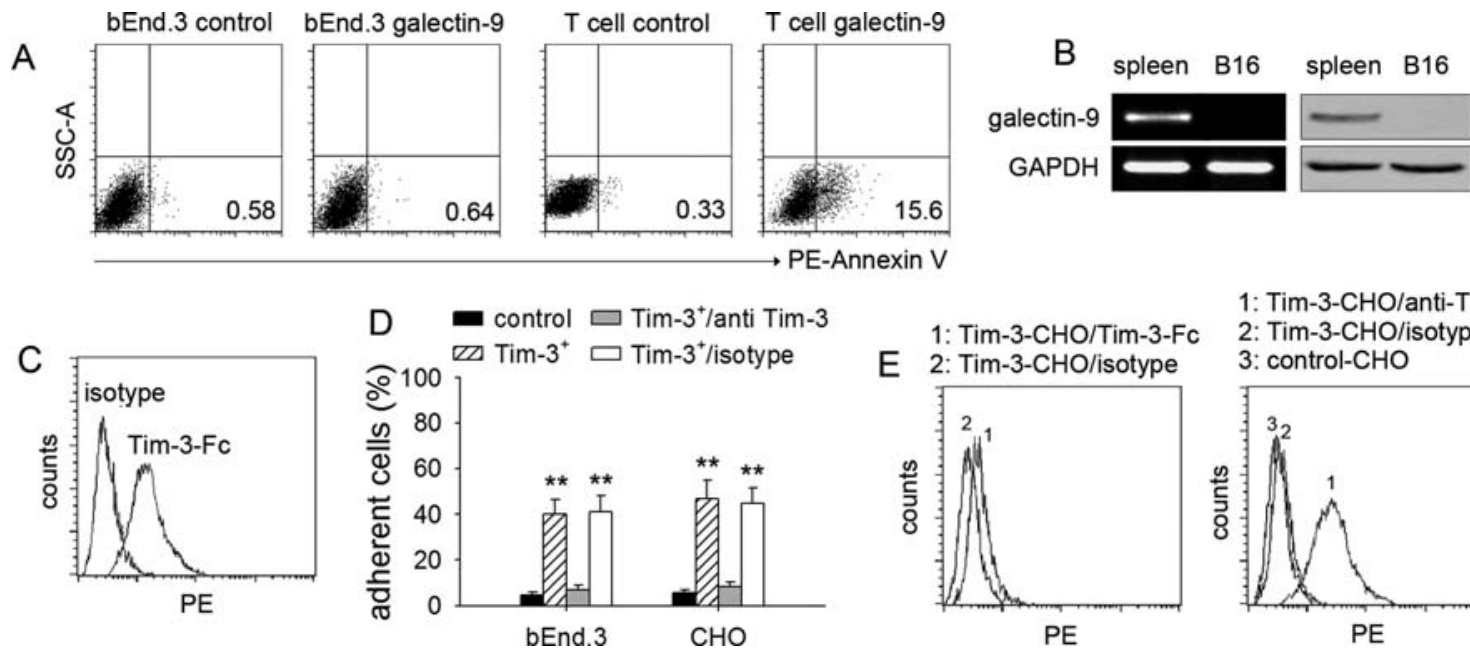

1: Tim-3-CHO/anti-Tim-3

2: Tim-3-CHO/isotype 3: control-CHO

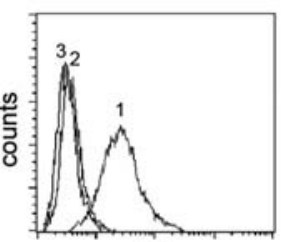

PE

Figure 2. Tim-3 mediates the interaction of endothelial cells with tumor cells. (A) Galectin-9 does not induce apoptosis of endothelial cells. The apoptosis of Tim- $3^{+}$bEnd.3 cells (left) was analyzed after 24-h culture in absence or presence of galectin-9. Con A-activated T cells were used as control (right). (B) Analysis of galectin-9 expression. Total RNA and proteins prepared from B16 cells were analyzed by RT-PCR and Western blotting, respectively. The spleen tissue was used as positive control. (C) Analysis of Tim-3-binding molecule on tumor cells. B16 cells were analyzed by flow cytometry with Tim-3-Fc. Isotype antibody was used as control. (D) Adhesion of B16 cells to Tim- $3^{+}$cells. B16 cells were incubated with pre-fixed Tim- $3^{+}$bEnd.3 or Tim-3-CHO cells in absence or presence of anti-Tim-3 antibody or isotype antibody. Both non-adherent cells and adherent cells were counted for the calculation of adhesion \%. ${ }^{* *} \mathrm{P}<0.01$, compared with control. (E) Identification of Tim-3-CHO cells. Tim-3-binding molecule (left) and Tim-3 (right) on the surface of cells were analyzed by flow cytometry.

The result showed that HMGB1 induced the expression of Tim-3 in bEnd.3 cells, which was suppressed by inhibiting NF- $\mathrm{KB}$ and AP-1, two transcription factors which can be activated by TLR4 signaling (Fig. 1E). Consistently, HMGB1 mainly induced Tim- 3 expression in TLR $4^{+}$bEnd. 3 cells (Fig. 1F). These data suggest that Tim-3 expression in endothelial cell can be induced by tumor cell-released TLR4 ligand.

Tim-3 mediates the interaction of endothelial cells with tumor cells. To explore the role of Tim-3 in endothelial cells, we first stimulated bEnd.3 cells with galectin 9, the ligand for Tim-3 which induces $\mathrm{T}$ cell apoptosis (9). The result showed that galectin-9 did not induce apoptosis of Tim- $3^{+}$ bEnd. 3 cells (Fig. 2A). Therefore, we explored the possibility that Tim-3 on endothelial cells may mediate the interaction of endothelial cells with tumor cells. Although galectin-9 was not detectable in B16 melanoma cells (Fig. 2B), a Tim-3binding molecule on the surface of B16 cells was detected by flow cytometry (Fig. 2C). Furthermore, B16 cells could adhere to Tim- $3^{+}$bEnd .3 cells, which was inhibited by anti-Tim-3 antibody (Fig. 2D), suggesting that Tim-3 and Tim-3-binding 

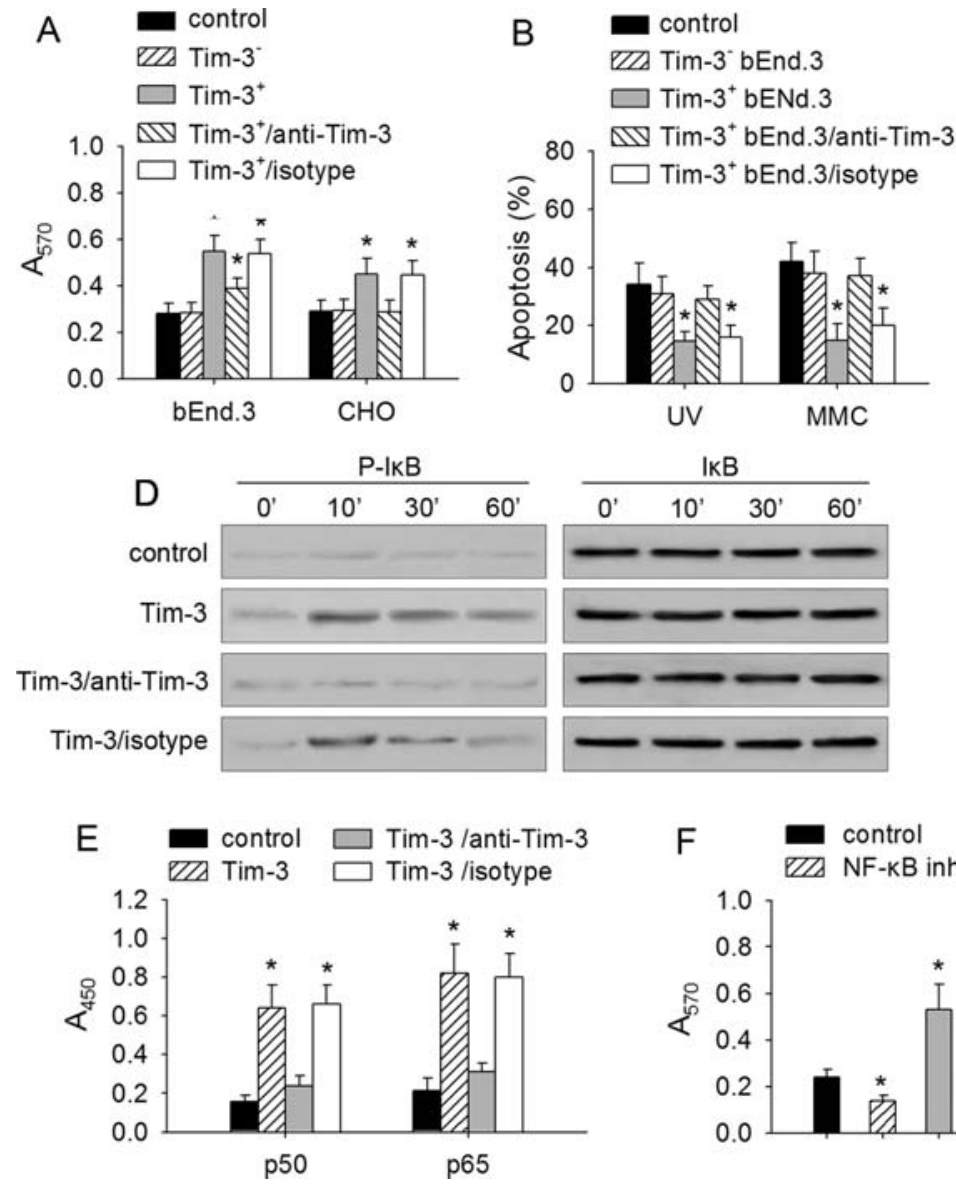

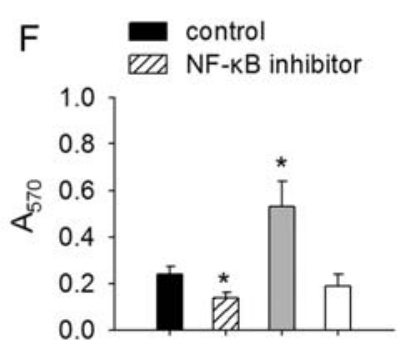

0.0
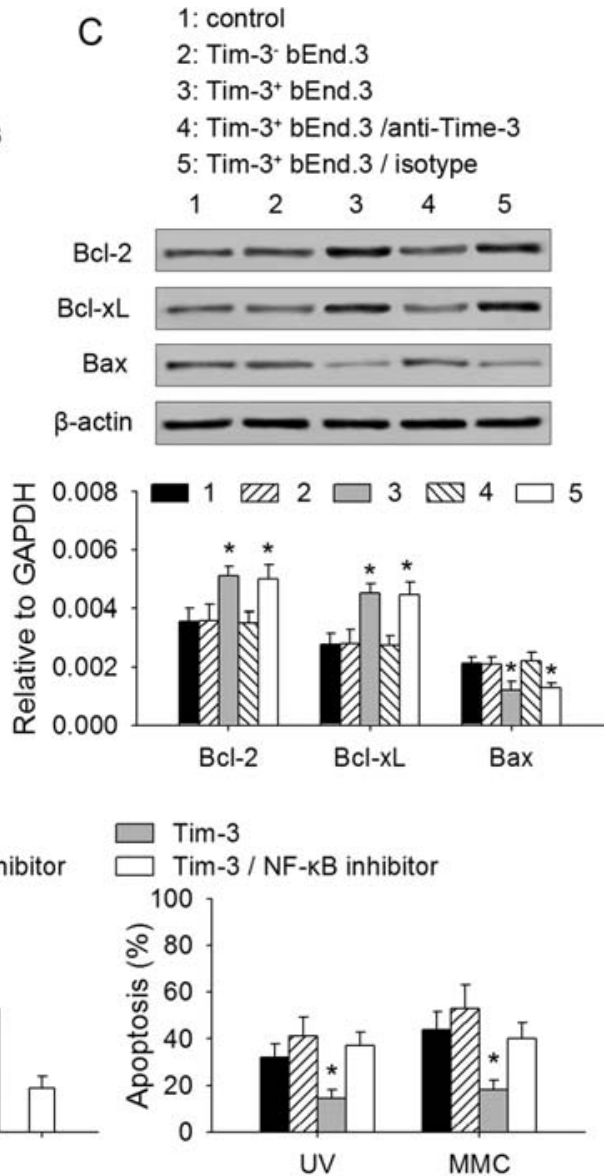

Figure 3. Tim-3 promotes tumor cell proliferation and apoptosis-resistance. (A) Effect of Tim-3 on tumor cell proliferation. B16 cells were cultured with or without pre-fixed corresponding Tim- $3^{-}$or Tim- $3^{+}$cells in absence or presence of anti-Tim-3 blocking antibody or control isotype for 48 h. The proliferation was determined with MTT Cell Proliferation kit. (B and C) Effect of Tim-3 on tumor cell apoptosis. B16 cells were stimulated with Tim- $3^{+}$bEnd.3 cells as described in (A) for $48 \mathrm{~h}$. The cells were then irradiated with UV or treated with MMC for $12 \mathrm{~h}$ for the analysis of apoptosis by flow cytometry (B) or used for the analysis of expressions of Bcl-2, Bcl- $x \mathrm{~L}$ and Bax genes by Western blotting (C, top) and real-time RT-PCR (C, bottom). (D and E) Analysis of Tim-3 signaling. B16 cells were stimulated with pre-fixed control-CHO cells or Tim-3-CHO in absence or presence of anti-Tim-3 antibody or control isotype. The

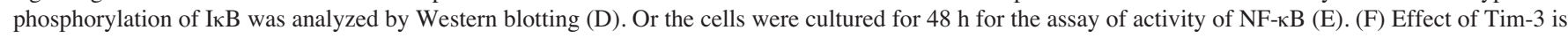

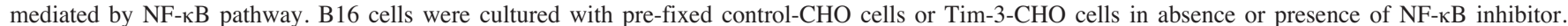
Tumor cell proliferation (left) and apoptosis \% (right) were analyzed as described in (A) and (B). ${ }^{*} \mathrm{P}<0.05$, compared with control in this figure.

molecule could mediate the interaction of endothelial cells with tumor cells. We then constructed a CHO cell line (Tim-3CHO) which expressed Tim-3 but not Tim-3-binding molecule (Fig. 2E). Tumor cells also adhered to Tim-3-CHO cells, which was inhibited by anti-Tim-3 antibody (Fig. 2D). Collectively, these data suggest that Tim-3, without cooperation with other molecule(s) expressed by endothelial cells, mediates the interaction of endothelial cells with tumor cells.

Tim-3 promotes tumor cell proliferation and apoptosisresistance through $N F-\kappa B$ pathway. We next investigated the effect of Tim-3 on tumor cells by culturing B16 cells in the presence of pre-fixed Tim- $3^{+}$cells. The result showed that Tim- $3^{+}$cells significantly increased the proliferation of B16 cells, which was inhibited by anti-Tim-3 antibody (Fig. 3A). In addition, Tim-3 also increased resistance of tumor cells to UV irradiation or treatment with MMC (Fig. 3B). Consistently, the expressions of the genes related to apoptosis were altered, including up-regulation of Bcl-2 and Bcl-xL and downregulation of Bax (Fig. 3C). We then used Tim-3-CHO cells as the donor of Tim-3 to stimulate tumor cells, and analyzed the $\mathrm{NF}-\kappa \mathrm{B}$ pathway which is important for tumor proliferation

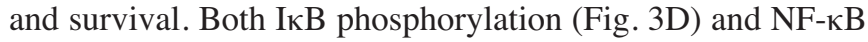
activity (Fig. 3E) in tumor cells were increased after stimulation with Tim-3, which was inhibited by anti-Tim-3 antibody. Consistently, the inhibition of NF- $\mathrm{NB}$ activity counteracted the effect of Tim-3 on proliferation and apoptosis of B16 cells (Fig. 3F). Therefore, Tim-3 can trigger signal transduction in tumor cells, leading to accelerated proliferation and enhanced apoptosis-resistance of tumor cells.

Endothelial cell-expressed Tim-3 promotes metastasis of B16 cells to the lung. We next investigated whether Tim-3 may facilitate metastasis of melanoma cells by using a model of experimental lung metastasis of melanoma cells. The stimulation with Tim- $3^{+}$bEnd .3 cells increased the resistance 


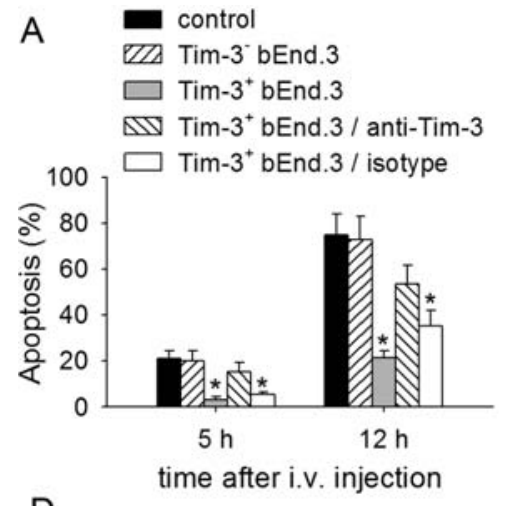

B
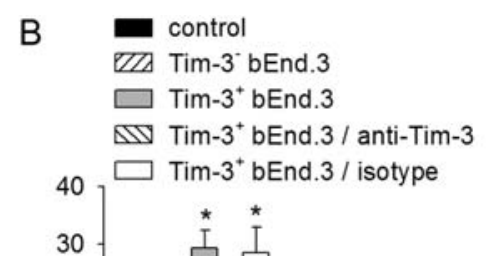

D

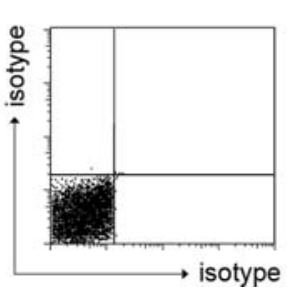

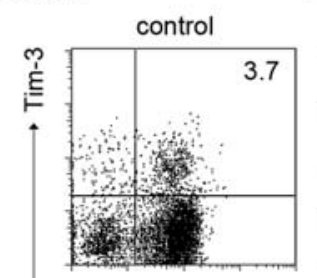

3.7

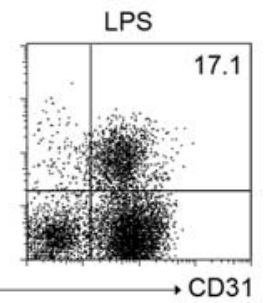

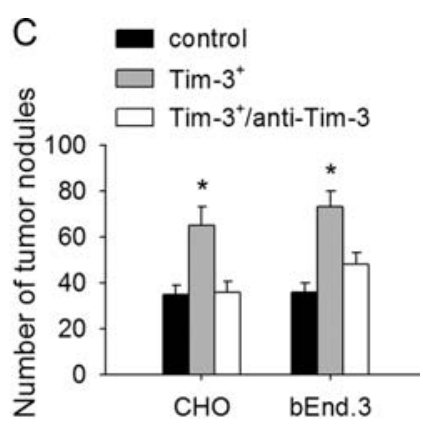

E

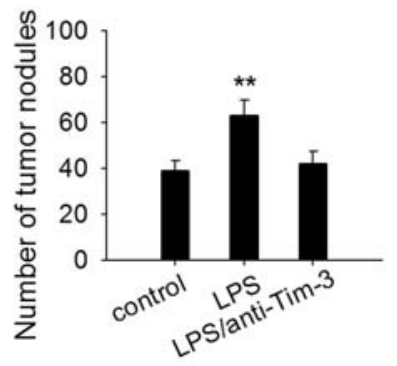

Figure 4. Endothelial Tim-3 promotes metastasis of B16 melanoma cells to lung. (A and B) Tim-3 enhances apoptosis-resistance of tumor cells in blood and tumor cell arrest in the lung. B16 cells were cultured under the same conditions as described in Fig. 3B, labeled with CFSE, and then injected into mice via tail vein. The proportion of apoptotic B16 cells in blood from mice ( $\mathrm{n}=8$ in each group) was analyzed 5 and $12 \mathrm{~h}$ later by flow cytometry (A). Tumor cell arrest and invasion were analyzed by counting fluorescent spots in the sections prepared from mice ( $\mathrm{n}=8$ in each group) 5 and $24 \mathrm{~h}$ after tumor cell injection (B). (C) The metastatic capability of B16 cells. B16 cells were treated with pre-fixed control or Tim- $3^{+}$cells in absence or presence anti-Tim-3 antibody and then injected to mice ( $n=8$ per group). The tumor nodules in lung were counted 15 days later. (D) Effect of LPS on Tim-3 expression in lung endothelial cells. Endothelial cells were isolated from the lungs of mice $24 \mathrm{~h}$ after the administration of LPS (10 $\mu \mathrm{g})$ aerosol. Tim-3 expression in endothelial cells was analyzed by flow cytometry. (E) Effect of Tim-3 expression in target organ on tumor metastasis. Mice (n=8 per group) were treated by intranasal administration of LPS aerosol. Seventy-two hours later, the mice were inoculated with B16 cells by i.v. injection with or without anti-Tim-3 antibody treatment. The tumor nodules in lung were counted 15 days after inoculation. ${ }^{*} \mathrm{P}<0.05$, compared with control in this figure.

of B16 cells to the shear force of blood in vivo (Fig. 4A), and also increased tumor cell arrest in the lung and following invasion into lung tissue, as evaluated by fluorescent spots both 5 and 24 h after i.v. injection of CFSE-labeled B16 cells (Fig. 4B). Consistently, Tim-3-stimulated B16 cells formed more metastatic nodules in lung after i.v. injection (Fig. 4C). Anti-Tim-3 antibody suppressed the effect of Tim-3 on B16 cells. These data implied that endothelial Tim-3 may facilitate tumor metastasis during tumor cell intravasation. We then analyzed the effect of endothelial Tim-3 on tumor cell extravasation. The expression of endothelial Tim-3 in the lung was increased by challenge with LPS aerosol (Fig. 4D). The increased expression of Tim-3 in endothelial cells resulted in more metastatic nodules in the lung after i.v. injection of B16 cells (Fig. 4E). The injection of anti-Tim-3 antibody suppressed the metastasis-promoting effect of LPS aerosolization (Fig. 4E). Thus, these data demonstrated that endothelium-expressed Tim-3 is beneficial to tumor metastasis.

\section{Discussion}

We previously showed that Tim-3 may act as a signal molecule to promote tumor progression (18). Here, we further provide evidence that Tim-3 expression in endothelial cells in tumor and lung could be induced by the TLR4 ligand. Tim-3 then mediates the interaction of endothelial cells with tumor cells, promoting tumor cell proliferation and metastasis.
TLR4 is the receptor for various inflammation-associated molecules, such as endotoxin (LPS) and dead cell-released HSPs and HMGB1 (20). Endothelial cells also express TLR4 (6). Therefore, endothelial cells can respond to TLR4 ligands, and release proinflammatory factors. In this study, we found that Tim-3 gene was induced in endothelial cells by TLR4 signaling. HMGB1 activated $\mathrm{NF}-\kappa \mathrm{B}$ and MAPK pathways which in turn cooperatively activated the expression of Tim-3. Inhibiting NF-кB and AP-1 activities suppressed the expression of Tim-3 in HMGB1-stimulated endothelial cells. Therefore, the endothelial cells in response to TLR4 ligand not only produce proinflammatory factors, but also express Tim-3 to communicate with tumor cells.

Tim-3 is primarily delineated by three basic points: Th1specific, inhibitory receptor, and signaling by ligation with galectin-9 $(8,9)$. Its function in other types of cells is still incompletely understood, although a correlation of Tim-3 expression with inflammation was implied recently $(11-13,21)$. Here, we show that endothelial cell-expressed Tim-3 does not function as an apoptosis-inducing receptor, but mediates the communication between endothelial cells and tumor cells. B16 melanoma cells do not express galectin 9, but express a membrane-associated molecule which binds Tim-3 and actually functions as receptor of Tim-3. The engagement of endothelial cell-expressed Tim-3 with its receptor on tumor cells could transduce a positive signal into tumor cells, resulting in the activation of NF- $\mathrm{KB}$ pathway in tumor cells. In the process of intravasation and extravasation, tumor cells 
may receive Tim-3 signaling from endothelial cells to increase their proliferative potential and apoptosis-resistance, thus benefiting tumor metastasis.

HMGB1 has been identified as a proinflammatory cytokine which can be secreted by different cells or released from necrotic cells (22-24). Higher HMGB1 levels have been found in tumors with greater metastatic potential (25). Except for inducing Tim-3 expression in endothelial cells as shown in this study, HMGB1 also up-regulates the expression of Eselectin in endothelial cells $(4,25)$. E-selectin modulates transmigration of tumor cells by enhancing transendothelial permeability and migration of tumor cells (26-28). Thus, endothelial E-selectin and Tim-3 might synergize to benefit tumor metastasis by promoting migration and survival of tumor cells.

Extravasation of tumor cells is a pivotal step in the formation of hematogenous metastasis. Tim-3-expressing endothelial cells may interact with tumor cells to potentiate metastasis by increasing proliferative potential and apoptosis-resistance of tumor cells. In line with this, our data showed that the increased Tim-3 expression in lung endothelial cells promoted the metastasis of circulating tumor cells. There could be a positive feedback regulation in the process of tumor metastasis. Dead tumor cells in blood release HMGB 1 which may induce E-selectin expression by endothelial cells. E-selectin in turn enhances the release of HMGB 1 from tumor cells when tumor cells interact with endothelial cells (25). Thus, the amount of HMGB1 may be further increased, leading to up-regulation of Tim-3 in endothelial cells. Therefore, our data imply that dead tumor cells in circulation may help the metastasis of living tumor cells by stimulating Tim-3 expression in endothelial cells.

In conclusion, our findings in this report have important implications in understanding the molecular basis of intercellular communication between endothelial cells and tumor cells. First, tumor cells release HMGB1 to stimulate endothelial cells as part of inflammation reactions. Second, Tim-3 expressed by endothelial cells mediates the signaling from endothelial cells to tumor cells. Third, the establishment of this communication in inflammation promotes tumor metastasis. The role of Tim-3 in tumor metastasis may not only provide new evidence for understanding the connection between the inflammatory response and cancer, but also may lead to the discovery of new therapeutic targets in cancer therapy.

\section{Acknowledgements}

This study was supported by National Development Program (973) For Key Basic Research (2009CB521806) of China and the National Natural Science Foundation of China (No. 30830095, No. 30772589).

\section{References}

1. Folkman J: Role of angiogenesis in tumor growth and metastasis. Semin Oncol 29: 15-18, 2002.

2. Hanahan D and Weinberg RA: The hallmarks of cancer. Cell 100: 57-70, 2000.

3. Pober JS and Sessa WC: Evolving functions of endothelial cells in inflammation. Nat Rev Immunol 7: 803-815, 2007.
4. Van Beijnum JR, Buurman WA and Griffioen AW: Convergence and amplification of toll-like receptor (TLR) and receptor for advanced glycation end products (RAGE) signaling pathways via high mobility group B1 (HMGB1). Angiogenesis 11: 91-99, 2008.

5. Chen LC, Gordon RE, Laskin JD and Laskin DL: Role of TLR-4 in liver macrophage and endothelial cell responsiveness during acute endotoxemia. Exp Mol Pathol 83: 311-326, 2007.

6. Fitzner N, Clauberg S, Essmann F, Liebmann J and KolbBachofen V: Human skin endothelial cells can express all 10 TLR genes and respond to respective ligands. Clin Vaccine Immunol 15: 138-146, 2008.

7. Meroni PL, Raschi E, Testoni C, Parisio A and Borghi MO: Innate immunity in the antiphospholipid syndrome: role of tolllike receptors in endothelial cell activation by antiphospholipid antibodies. Autoimmun Rev 3: 510-515, 2004.

8. Kuchroo VK, Meyers JH, Umetsu DT and DeKruyff RH: TIM family of genes in immunity and tolerance. Adv Immunol 91: 227-249, 2006.

9. Zhu C, Anderson AC, Schubart A, Xiong H, Imitola J, Khoury SJ, Zheng XX, Strom TB and Kuchroo VK: The Tim-3 ligand galectin- 9 negatively regulates $\mathrm{T}$ helper type 1 immunity. Nat Immunol 6: 1245-1252, 2005.

10. Van de Weyer PS, Muehlfeit M, Klose C, Bonventre JV, Walz G and Kuehn KW: A highly conserved tyrosine of Tim-3 is phosphorylated upon stimulation by its ligand galectin-9. Biochem Biophys Res Commun 351: 571-576, 2006.

11. Wiener Z, Kohalmi B, Pocza P, Jeager J, Tolgyesi G, Toth S, Gorbe E, Papp Z and Falus A: TIM-3 is expressed in melanoma cells and is up-regulated in TGF-beta stimulated mast cells. J Invest Dermatol 127: 906-914, 2007.

12. Oikawa T, Kamimura Y, Akiba H, Yagita H, Okumura K, Takahashi H, Zeniya M, Tajiri H and Azuma M: Preferential involvement of Tim-3 in the regulation of hepatic $\mathrm{CD}^{+} \mathrm{T}$ cells in murine acute graft-versus-host disease. J Immunol 177: 4281-4287, 2006.

13. Renesto PG, Ponciano VC, Cenedeze MA, Saraiva Câmara NO and Pacheco-Silva A: High expression of Tim-3 mRNA in urinary cells from kidney transplant recipients with acute rejection. Am J Transplant 7: 1661-1665, 2007.

14. Frisancho-Kiss S, Davis SE, Nyland JF, Frisancho JA, Cihakova D, Barrett MA, Rose NR and Fairweather D: Cutting edge: cross-regulation by TLR4 and T cell Ig mucin- 3 determines sex differences in inflammatory heart disease. J Immunol 178: 6710-6714, 2007.

15. Kearley J, McMillan SJ and Lloyd CM: Th2-driven, allergeninduced airway inflammation is reduced after treatment with anti-Tim-3 antibody in vivo. J Exp Med 204: 1289-1294, 2007.

16. Huang B, Pan PY, Li Q, Sato AI, Levy DE, Bromberg J, Divino CM and Chen SH: Gr- $1^{+} \mathrm{CD} 115^{+}$immature myeloid suppressor cells mediate the development of tumor-induced $\mathrm{T}$ regulatory cells and T-cell anergy in tumor-bearing host. Cancer Res 66: 1123-1131, 2006.

17. Gong W, Liu Y, Huang B, Lei Z, Wu FH, Li D, Feng ZH and Zhang GM: Recombinant CBD-HepII polypeptide of fibronectin inhibits alphavbeta3 signaling and hematogenous metastasis of tumor. Biochem Biophys Res Commun 367: 144$149,2008$.

18. Geng H, Zhang GM, Li D, Zhang H, Yuan Y, Zhu HG, Xiao H, Han LF and Feng ZH: Soluble form of T cell Ig mucin 3 is an inhibitory molecule in $\mathrm{T}$ cell-mediated immune response. $\mathrm{J}$ Immunol 176: 1411-1420, 2006.

19. Wada J, Ota K, Kumar A, Wallner EI and Kanwar YS: Developmental regulation, expression and apoptotic potential of galectin-9, a beta-galactoside binding lectin. J Clin Invest 99: 2452-2461, 1997.

20. Huang B, Zhao J, Unkeless JC, Feng ZH and Xiong H: TLR signaling by tumor and immune cells: a double-edged sword. Oncogene 27: 218-224, 2008.

21. Anderson AC, Anderson DE, Bregoli L, Hastings WD, Kassam N, Lei C, Chandwaskar R, Karman J, Su EW, Hirashima M, Bruce JN, Kane LP, Kuchroo VK and Hafler DA: Promotion of tissue inflammation by the immune receptor Tim-3 expressed on innate immune cells. Science 318: 1141-1143, 2007.

22. Fiuza C, Bustin M, Talwar S, Tropea M, Gerstenberger E, Shelhamer JH and Suffredini AF: Inflammation-promoting activity of HMGB1 on human microvascular endothelial cells. Blood 101: 2652-2660, 2003. 
23. Lotze MT, Zeh HJ, Rubartelli A, Sparvero LJ, Amoscato AA Washburn NR, Devera ME, Liang X, Tör M and Billiar T: The grateful dead: damage-associated molecular pattern molecules and reduction/oxidation regulate immunity. Immunol Rev 220: 60-81, 2007

24. Raucci A, Palumbo R and Bianchi ME: HMGB1: a signal of necrosis. Autoimmunity 40: 285-289, 2007.

25. Aychek T, Miller K, Sagi-Assif O, Levy-Nissenbaum O, IsraeliAmit M, Pasmanik-Chor M, Jacob-Hirsch J, Amariglio N, Rechavi G and Witz IP: E-selectin regulates gene expression in metastatic colorectal carcinoma cells and enhances HMGB1 release. Int J Cancer 123: 1741-1750, 2008.
26. Laferriere J, Houle F, Taher MM, Valerie K and Huot J: Transendothelial migration of colon carcinoma cells requires expression of E-selectin by endothelial cells and activation of stressactivated protein kinase-2 (SAPK2/p38) in the tumor cells. J Biol Chem 275: 33762-33772, 2001.

27. Laferriere J, Houle F and Huot J: Adhesion of HT-29 colon carcinoma cells to endothelial cells requires sequential events involving E-selectin and integrin beta4. Clin Exp Metastasis 21: 257-264, 2004

28. Tremblay PL, Auger FA and Huot J: Regulation of transendothelial migration of colon cancer cells by E-selectinmediated activation of p38 and ERK MAP kinases. Oncogene 25: 6563-6573, 2006. 